\title{
Association of mitochondrial respiratory chain enzymes with the risk and mortality of sepsis among Chinese children
}

Danni He ${ }^{1,2+}$, Ning Li ${ }^{3+}$, Xiuxiu Lu ${ }^{3}$, Wei Li ${ }^{3}$, Yuanmei Chen ${ }^{1}$, Zhongyuan Sun ${ }^{3}$, Lipeng Zhang ${ }^{2,4}$, Linying Guo ${ }^{3}$, Xiaodai Cui ${ }^{5}$, Guowei Song ${ }^{5}$, Wenquan $\mathrm{Niu}^{1 *}$ and Qi Zhang ${ }^{2^{*}}$ (])

\begin{abstract}
Background: Sepsis is a leading cause of pediatric morbidity and mortality worldwide. The aim of this study was to explore the association of decreased mitochondrial respiratory chain enzyme activities with the risk for pediatric sepsis, and explore their association with mortality among affected children.

Methods: A total of 50 incident cases with sepsis and 49 healthy controls participated in this study. The level of serum coenzyme Q10 was measured by high-performance liquid chromatography, and selected mitochondrial respiratory chain enzymes in WBC were measured using spectrophotometric. Logistic regression models were used to estimate odds ratio (OR) and 95\% confidence interval (Cl).

Results: The levels of CoQ10, complex II, complex I + III and FoF1-ATPase were significantly higher in healthy controls than in children with sepsis $(p<0.001,=0.004,<0.001$ and $<0.001$, respectively). In children with sepsis, levels of CoQ10 and complex I+ III were significantly higher in survived cases than in deceased cases $(p<0.001)$. Per $0.05 \mu \mathrm{mol} / \mathrm{L}, 50 \mathrm{nmol} / \mathrm{min} . \mathrm{mg}$ and $100 \mathrm{nmol} / \mathrm{min} . \mathrm{mg}$ increment in CoQ10, complex I+ III and FoF1-ATPase were associated with significantly lowered risk of having sepsis, even after adjusting for confounding factors $(\mathrm{OR}=0.85$, 0.68 and $0.04, p=0.001,<0.001$ and $<0.001$, respectively). Per $0.05 \mu \mathrm{mol} / \mathrm{L}$ and $50 \mathrm{nmol} / \mathrm{min}$.mg increment in CoQ10 and complex I+ III was associated with significantly lowered risk of dying from sepsis during hospitalization, and significance retained after adjustment $(\mathrm{OR}=0.73$ and $0.76,95 \% \mathrm{Cl}: 0.59$ to 0.90 and 0.64 to $0.89, p=0.004$ and 0.001 , respectively) in children with sepsis.
\end{abstract}

Conclusions: Our findings indicate the promising predictive contribution of low serum CoQ10 and complex I+ III to the risk of pediatric sepsis and its associated mortality during hospitalization among Chinese children.

Trial registration The trial was registered with www.chictr.org.cn, number ChiCTR-IOR-15006446 on May 05, 2015. Retrospectively registered.

Keywords: Sepsis, Children, Mitochondrial Respiratory Chain Enzymes, Risk, Mortality

*Correspondence: niuwenquan_shcn@163.com; zhangqikeyan@163.com ${ }^{\dagger}$ Danni He and Ning Li are first authors

${ }^{1}$ Institute of Clinical Medical Sciences, China-Japan Friendship Hospital, No. 2 Yinghua East Street, Chaoyang District, Beijing 100029, China

${ }^{2}$ Department of Pediatrics, China-Japan Friendship Hospital, No.2

Yinghua East Street, Chaoyang District, Beijing 100029, China

Full list of author information is available at the end of the article

\section{Background}

Sepsis is a leading cause of morbidity and mortality in children worldwide, with the case-fatality rate of $31.7 \%$ in developing countries and $19.3 \%$ in developed countries $[1,2]$. In China, the incidence rate of pediatric sepsis was estimated to be $181 / 100,000$ in 2014 [3]. The resolution of the World Health Assembly in 2017 has stressed the 
importance of developing more tools for sepsis diagnosis and treatment [4]. Current diagnostic strategies for identifying patients with sepsis mainly rely on clinical manifestations and markers of end-organ dysfunction, and the latest research has found that heat-shock-protein (HSP) $90 \alpha$ and human glucocorticoid receptor are also closely related to the death rate or severity of illness [5-7]. Since the definition, guidelines, biomarkers and pathways are constantly being improved and updated, early detection of sepsis using powerful tools or sensitive biomarkers and individualized close monitoring of patients at risk are of clinical and public health importance $[8,9]$.

Mitochondrial dysfunction is a feature of many pathologies, including sepsis [10-12]. Recent studies have shown a sharp decline in mitochondrial respiration of peripheral blood mononuclear cells in children with sepsis [13]. Moreover, mitochondrial dysfunction is also associated with kidney and liver injuries in patients with sepsis [14-16]. Besides, animal studies have shown that the reduction of mitochondrial calcium uptake was associated with the survival rate of rats [17]. Currently, most mitochondrion-sepsis correlation studies have focused on mitochondrial respiration or mitochondrial DNA $[18,19]$, yet few studies examined the enzymes and complexes on respiratory chains.

To fill this gap in knowledge and yield more information for future studies, we enrolled children suffering sepsis and healthy controls and assayed the activities of major mitochondrial respiratory chain enzymes in circulation, aiming to explore the association of mitochondrial respiratory chain enzyme activities with the risk for pediatric sepsis, and explore their association with hospitalized mortality among affected children.

\section{Methods}

\section{Study Subjects}

All study subjects were recruited from the Children's Hospital Affiliated to the Capital Institute of Pediatrics during the period from March 2013 to August 2014. This study received approval from the Ethics Committee of this hospital, and was conducted in compliance with the Declaration of Helsinki. This study complied with the STROBE standard as was listed in Additional file 1: eFile S1.

A total of 50 children who were clinically confirmed to have sepsis were classified as the case group, and 49 age- and sex-matched healthy controls who had no signs of sepsis formed the control group. Healthy controls were from those undergoing health check-ups at the health clinic in our hospital. All study subjects in control group had no physical discomfort and abnormal indexes in blood routine or biochemical examinations.

\section{Tissue collection and diagnosis}

Children aged 1 month to 192 months who were admitted to the Pediatric Intensive Care Unit (PICU) diagnosed with sepsis (Sepsis was SIRS in the presence of or as a result of suspected or proven infection. Severe Sepsis was defined as the presence of two or more systemic inflammatory response syndrome criteria, documented or suspected infection, and evidence of sepsis-induced organ dysfunction or tissue hypoperfusion as defined by International pediatric sepsis consensus conference: Definitions for sepsis and organ dysfunction in pediatrics [20]) were consecutively enrolled. For the definition of sepsis, please see Additional file 2: eFile S2. Children were excluded due to the following reasons: (i) taking medications affecting mitochondrial function, such as adenosine, ATP, CoQ10 inhibitors or modeling inhibitors; (ii) using immunosuppressant or immunomodulator within 4 weeks; (iii) congenital organ dysfunction; (iv) congenital and acquired immunodeficiency diseases; (v) not being of Han nationality. The participant flow diagram was shown in Fig. 1.

\section{Baseline characteristics and clinical biomarkers}

Baseline information was abstracted from the medical record system. Blood samples were collected from all affected children in the first $24 \mathrm{~h}$ of admission and stored with ethylenediaminetetraacetic acid (EDTA) at $4^{\circ} \mathrm{C}$. Then blood samples were centrifuged at $2000 \mathrm{rpm}$ for $10 \mathrm{~min}$ to separate the serum frozen at $-80{ }^{\circ} \mathrm{C}$ within $4 \mathrm{~h}$.

The following markers were assayed from blood samples: complete blood count, electrolytes, lymphocyte cells, IgM, IgA, IgG, C-Reactive Protein (CRP), Procalcitonin (PCT), serum coenzyme Q10 level (using highperformance liquid chromatography), Laboratories of BIOTECNOVO (Methanol (HPLC) Sigma-Aldrich America), and selected mitochondrial respiratory chain enzymes in WBC measured (using spectrophotometric method, Laboratories of BIOTECNOVO, SigmaAldrich America). Pediatric Critical Illness Score (PCIS) was tested at the time of the first diagnosis of sepsis, and vital status was determined at the time of discharge from PICU. Blood samples of healthy controls were collected on the date of physical examination. Serum coenzyme Q10 concentration levels and mitochondrial complex activities were assayed at the Institute of Experimental Science, and according to the signed informed consent form, children in the healthy group only extracted these indicators.

As mentioned earlier, it is used high-performance liquid Chromatography to measure CoQ10 levels [21]. In a nutshell, $25 \mu \mathrm{L}$ of ethanol solution included $12.5 \mathrm{ng}$ CoQ9 (used as internal standard) in an eppendorf tube 


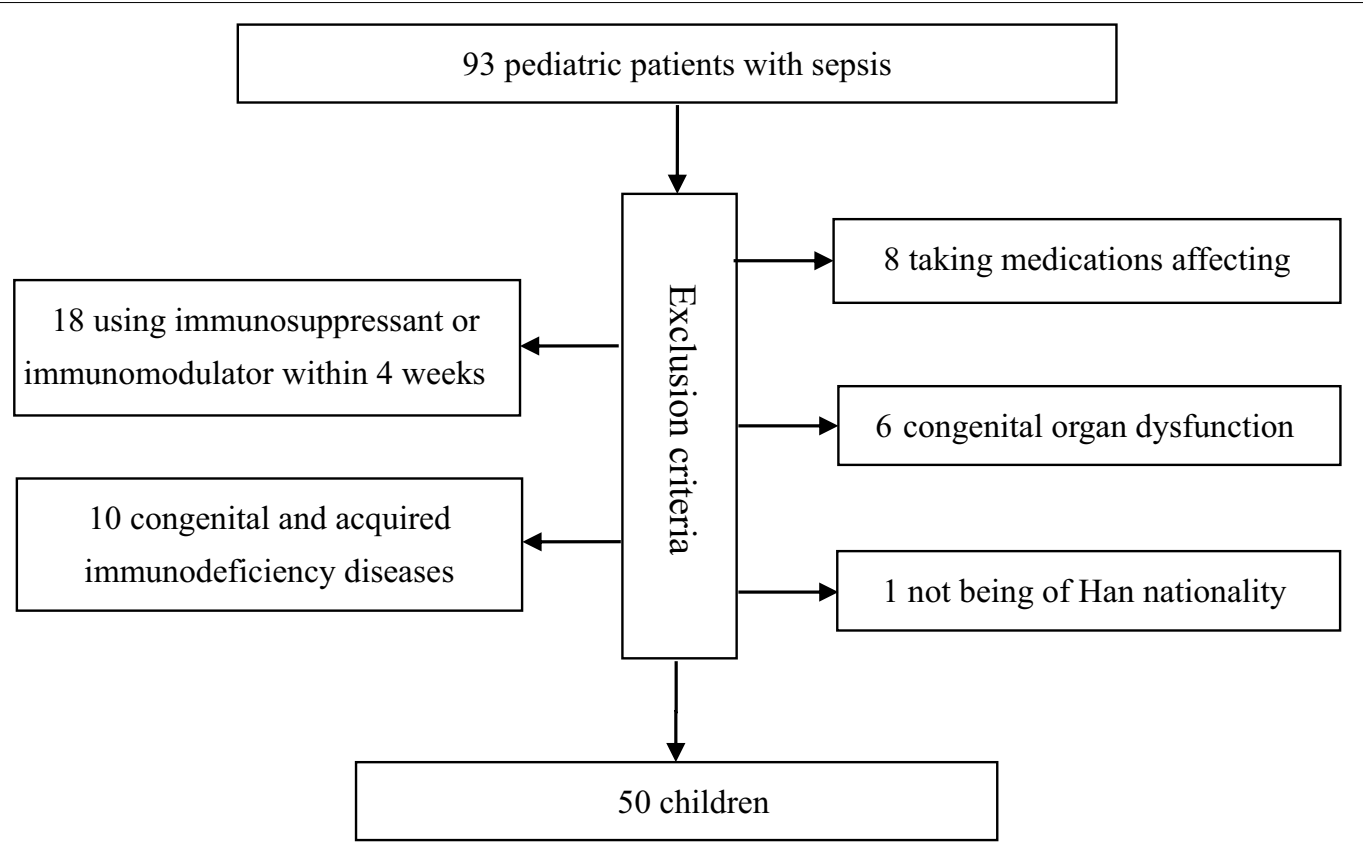

Fig. 1 Flow diagram of included participants

was blended wit $25 \mu \mathrm{L}$ of defrosted plasma. After adding $450 \mu \mathrm{L}$ 1-Propanol, the tube vortexes were mixed for $2 \mathrm{~min}$, then the high-speed was centrifuge in a cold room for $10 \mathrm{~min}$. The divisible $(\sim 300 \mu \mathrm{L})$ superclear is filtered by a $0.2 \mu \mathrm{m}$ filter column and transferred to an HPLC injection flask and a divisible $50 \mu \mathrm{L}$ extract injected into an HPLC system equipped with a C18 reversed phase column and ESA Coulochem II electrochemical detector(ESA, Inc., Chelmsford, MA).In our study measured the plasma CoQ10 in 49 age- and sexmatched healthy controls, and discovered the mid-value of $0.88 \mu \mathrm{mol} \mathrm{L}-1$ (IQR $0.82-0.95$ ), which is similar to the establishment of health control ranges in the literature $(1.04 \pm 0.33 \mu \mathrm{mol} \mathrm{L}-1)$.

Once plasma was collected, peripheral blood mononuclear cells (PBMCs) were segregated by high speed centrifuge [22]. PBMCs were used to measure mitochondrial, apoptotic, and oxidative stress markers; fresh cells were used to measure mitochondrial membrane potential and oxygen consumption, and frozen cells were used for the remaining measures. All PBMCs were normalized to protein content measured according to the Bradford protein dye binding based method [23].The enzymatic mitochondrial complexes were measured by thermostat zed spectrophotometry at $37{ }^{\circ} \mathrm{C}$ according to the methodology of Rustin et al. [24], slightly modified for complex IV measurement in minute amounts of biological samples [25]. Measurement the cells requiring treatment prior to complex I and triton activities, digitalis saponin detergent was used to obtain specific mitochondrial NADH-dehydrogenase activity [26]. Mitochondrial respiratory chain enzyme was represented as nanomoles substrate or produce products that consume and milligramme of protein per minute.

\section{Statistical analyses}

The $X^{2}$ tests for categorical data and Wilcoxon rank-sum tests for continuous data were used to assess the baseline characteristics among deceased and survived in patients with sepsis, as well as between survived and deceased children during hospitalization. Logistic regression analyses were conducted to assess the association of serum coenzyme Q10 and mitochondrial complex activities with the risk and mortality risk of sepsis at a significance level of $5 \%$ before and after adjusting for age and gender. Effect size estimates are expressed as odds ratio (OR) and 95\% confidence interval (95\% CI).

To test the performance of Logistic regression model, statistical indexes from calibration and discrimination aspects were adopted. Calibration capability was evaluated using the -2 log-likelihood ratio test, Akaike information criterion (AIC), and Bayesian information criterion (BIC) [27] to see how closely the prediction probability for the addition of serum coenzyme Q10 and mitochondrial complex activities reflected the actual observed risk and the global fit of modified risk model. Net reclassification improvement (NRI) and integrated discrimination improvement (IDI) [28, 29] were 
calculated to judge the discrimination capability of serum coenzyme Q10 and mitochondrial complex activities. Receiver operating characteristic (ROC) curves [30] were plotted based on the basic model and the plus of each enzyme detection index.

Finally, a prediction nomogram model was constructed based on factors of significance using the "rms" package in the open-source R software, version 3.5.1 (available at the website: https://www.r-project.org).

Unless otherwise indicated, STATA software Release 14.1 (Stata Corp, TX, USA) was used for statistical analyses. A P value of less than 0.05 was considered statistically significant, and for multiple comparisons, Bonferroni correction method was used.

\section{Results}

\section{Baseline characteristics}

This study included 50 children with sepsis with an average age of 27.21 months, including $36(72.0 \%)$ males. The control group contained 49 gender, age-matched healthy children with an average age of 26.72 months, containing $35(71.4 \%)$ males $(\mathrm{P}>0.05)$. The baseline characteristics between deceased and survived children with sepsis are presented in Table 1.

Distributions and comparisons of the activities of mitochondrial respiratory chain enzymes between healthy controls and children with sepsis, as well as between deceased and survived children during hospitalization, are shown in Table 2. The levels of CoQ10, complex II, complex I+III and FoF1-ATPase were significantly higher in controls than in cases $(\mathrm{P}:<0.001,0.004,<0.001$ and $<0.001$, respectively). In children with sepsis, levels of CoQ10 and complex I+ III were significantly higher in survived cases than in deceased cases (both $\mathrm{P}<0.001$ ).

\section{Sepsis risk and mortality risk}

The association of mitochondrial respiratory chain enzymes with sepsis development and mortality is shown in Table 3 before and after confounding adjustment. Per $0.05 \mu \mathrm{mol} / \mathrm{L}, 50 \mathrm{nmol} / \mathrm{min} . \mathrm{mg}$ and $100 \mathrm{nmol} / \mathrm{min} . \mathrm{mg}$ increment in CoQ10, complex I+ III and FoF1-ATPase (a rotary molecular motor driven by ATP-hydrolysis) were associated with significantly lowered risk of having sepsis, even after adjusting for age and sex $(\mathrm{OR}=0.85,0.68$ and $0.53, \mathrm{P}: 0.001,<0.001$ and $<0.001$, respectively), yet the association of complex II and complex II + III with sepsis risk was only marginally significant.

In children with sepsis, per $0.05 \mu \mathrm{mol} / \mathrm{L}$ and $50 \mathrm{nmol} /$ min.mg in CoQ10 and complex I+ III was associated with significantly lowered risk of dying from sepsis during hospitalization, and significance retained after controlling for age and sex $(\mathrm{OR}=0.73$ and $0.76,95 \%$ CI: 0.59 to 0.90 and 0.64 to $0.89, \mathrm{P}=0.004$ and 0.001 , respectively). No signs of significance were noted for the other biomarkers.

\section{Accuracy appraisal of mortality prediction}

The prediction accuracy gained by adding mitochondrial respiratory chain enzymes individually to the basic model is summarized in Table 4. As reflected by both calibration and discrimination statistics, only CoQ10 and complex I+ III exhibited significant contribution to the mortality risk of hospitalized children with sepsis. Prediction accuracy was reinforced after adding both biomarkers simultaneously to the basic model.

\section{Nomogram prediction model}

In light of the significant contribution of low serum CoQ10 and complex I+III to mortality risk, a nomogram prediction model incorporating age, PCIS, CoQ10 and complex I+ III was constructed (Fig. 2). The accuracy of this model reached as high as $92.3 \%(\mathrm{P}<0.001)$.

\section{Discussion}

The aim of this study was to explore the association of mitochondrial respiratory chain enzyme activities with the risk of sepsis and its associated mortality risk among hospital-based children. The key findings are the promising predictive contribution of low serum CoQ10 and complex I+ III levels to the risk of pediatric sepsis and its associated mortality during hospitalization, highlighting the importance of mitochondrial respiratory chain enzymes in the development and progression of pediatric sepsis. To the best of our knowledge, this is the first study that has evaluated the association of circulating mitochondrial respiratory chain enzyme activities with sepsis risk in the literature.

Currently, the biological implications of mitochondrial dysfunction in sepsis have been widely evaluated [10, 31, 32]. Mitochondria produce ATP by transferring electrons from substrates sequentially across four respiratory chain complexes (I to IV) and two mobile carriers (coenzyme Q and cytochrome C) to final electron acceptors [33]. Mitochondrial dysfunction is deemed as a key cellular event involved in the pathogenesis of multi-organ failure in sepsis, and it is secondary to tissue hypoxia and involves various toxins or mediators of inflammation that impair oxygen utilization (cytopathic hypoxia) [33, 34]. There is evidence that damaged mitochondria contribute to NACHT, LRR and PYD domains-containing protein 3 (NLRP3) inflammasome-related sepsis [31]. In addition, hydrogen was found to alleviate mitochondrial dysfunction and cytokine release via autophagy-mediated NLRP3 inflammasome inactivation [13]. On the basis of above evidence, it is reasonable to hypothesize that 
Table 1 Baseline characteristics of study participants in this study

\begin{tabular}{|c|c|c|c|}
\hline Characteristics & Deceased & Survived & $P$ \\
\hline Age, months & $26 \pm 37.13$ & $27.32 \pm 35.23$ & 0.835 \\
\hline Male, n (\%) & $11(68.8 \%)$ & $25(73.5 \%)$ & 0.726 \\
\hline SOFA, n (\%) & & & 0.047 \\
\hline 0 to 10 & $3(18.8 \%)$ & $19(55.9 \%)$ & \\
\hline 10 to 20 & $11(68.8 \%)$ & $13(38.2 \%)$ & \\
\hline$>20$ & $2(12.5 \%)$ & $2(5.9 \%)$ & \\
\hline PCIS, n (\%) & & & 0.005 \\
\hline 80 to 100 & $2(12.5 \%)$ & $19(55.9 \%)$ & \\
\hline 71 to 80 & $4(25 \%)$ & $8(23.5 \%)$ & \\
\hline 0 to 70 & $10(62.5 \%)$ & $7(20.6 \%)$ & \\
\hline MODS > 4 Organs, n (\%) & $14(87.5 \%)$ & $14(41.2 \%)$ & 0.002 \\
\hline MOF > 3 Organs, n (\%) & $11(68.8 \%)$ & $9(26.5 \%)$ & 0.004 \\
\hline $\mathrm{DIC}, \mathrm{n}(\%)$ & $14(87.5 \%)$ & $22(64.7 \%)$ & 0.094 \\
\hline Shock, n (\%) & $13(81.3 \%)$ & $23(67.6 \%)$ & 0.318 \\
\hline ARDS, n (\%) & $12(75 \%)$ & $19(55.9 \%)$ & 0.194 \\
\hline Brain Failure, $\mathrm{n}(\%)$ & $14(87.5 \%)$ & $21(61.8 \%)$ & 0.064 \\
\hline Respiratory Failure, n (\%) & $13(81.3 \%)$ & $26(76.5 \%)$ & 0.704 \\
\hline Heart Failure, $n(\%)$ & $11(68.8 \%)$ & $8(23.5 \%)$ & 0.002 \\
\hline Liver Failure, n (\%) & $12(75 \%)$ & $6(17.6 \%)$ & $<0.001$ \\
\hline Kidney Failure, n (\%) & $6(37.5 \%)$ & $5(14.7 \%)$ & 0.07 \\
\hline Stomach Failure, n (\%) & $12(75 \%)$ & $14(41.2 \%)$ & 0.026 \\
\hline Pancreas Failure, n (\%) & $3(18.8 \%)$ & $1(2.9 \%)$ & 0.055 \\
\hline Metabolic Acidosis, n (\%) & $12(75 \%)$ & $16(47.1 \%)$ & 0.063 \\
\hline Th1 cell $(n / \mu l)$ & $4.2(1.9$ to 6.5$)$ & 2.3 (1.9 to 2.6$)$ & 0.308 \\
\hline Th2 cell $(n / \mu l)$ & 0.3 (0.1 to 0.6$)$ & 0.1 (0.1 to 0.2$)$ & 0.075 \\
\hline CD3 $(n / \mu l)$ & 60 (53 to 71$)$ & 60 (40 to 73 ) & 0.721 \\
\hline CD4 $(n / \mu l)$ & 32.5 (22 to 44$)$ & 23 (17 to 41$)$ & 0.251 \\
\hline CD8 $(n / \mu l)$ & 21.5 (17 to 27 ) & 23 (18 to 33 ) & 0.818 \\
\hline CD4/CD8 & 1.55 (0.87 to 2.19 ) & $1(0.68$ to 1.5$)$ & 0.284 \\
\hline $\operatorname{CD} 19(n / \mu l)$ & 27.4 (24.4 to 41$)$ & 28 (9 to 46) & 0.858 \\
\hline CD16/CD56 & 6.5 (3 to 13$)$ & 9 (3 to 22$)$ & 0.294 \\
\hline $\lg G(g / L)$ & $8.86(6.46$ to 13.76$)$ & $14.47(10.26$ to 23.61$)$ & 0.039 \\
\hline $\lg A(g / L)$ & 0.8 (0.31 to 1.28$)$ & 0.36 (0.19 to 0.81 ) & 0.260 \\
\hline $\lg M(g / L)$ & 0.84 (0.53 to 1.09 ) & 0.53 (0.35 to 0.76$)$ & 0.061 \\
\hline TRIG (mmol/L) & 1.51 (0.97 to 2.39 ) & $2.22(1.5$ to 2.69$)$ & 0.105 \\
\hline $\mathrm{HDL}(\mathrm{mmol} / \mathrm{L})$ & 0.81 (0.59 to 1.03 ) & 0.69 (0.41 to 0.96$)$ & 0.173 \\
\hline $\mathrm{LDL}(\mathrm{mmol} / \mathrm{L})$ & 2.21 (1.63 to 2.79$)$ & 3.1 (2.17 to 3.79 ) & 0.038 \\
\hline $\mathrm{ALT}(\mathrm{U} / \mathrm{L})$ & $28(17.45$ to 68.55$)$ & 168.5 (37.3 to 181.1) & 0.003 \\
\hline AST (U/L) & 50.15 (30.95 to 121.9) & 104.1 (65.45 to 188.2$)$ & 0.105 \\
\hline$L D(U / L)$ & 320 (260 to 828$)$ & 874 (660 to 1089) & 0.016 \\
\hline $\mathrm{HBDH}(\mathrm{U} / \mathrm{L})$ & 293.5 (199 to 741$)$ & 539 (413 to 749$)$ & 0.118 \\
\hline CK (U/L) & 44.5 (32 to 77.5$)$ & 59 (20 to 142$)$ & 0.836 \\
\hline CKMB (ng/mL) & 18 (12 to 27$)$ & 23.5 (18 to 38 ) & 0.075 \\
\hline Glucose (mmol/L) & 5.61 (4.81 to 6.74 ) & 5.6 (3.2 to 8.15 ) & 0.639 \\
\hline $\mathrm{Ca}(\mathrm{mmol} / \mathrm{L})$ & 2.26 (2.08 to 2.35$)$ & 1.96 (1.81 to 2.28 ) & 0.015 \\
\hline WBC $\left(10^{\wedge} 9 / L\right)$ & 12.9 (8.14 to 16.65$)$ & 11.8 (7.02 to 16.96$)$ & 0.920 \\
\hline $\mathrm{CRP}(\mathrm{mg} / \mathrm{mL})$ & 8 (2 to 54$)$ & 23 (15 to 34$)$ & 0.116 \\
\hline PCT (ng/mL) & 1.38 (0.27 to 2.55$)$ & 1.7 (0.1 to 2.89 ) & 0.958 \\
\hline
\end{tabular}

P values are calculated by nonparametric Wilcoxon rank sum tests for continuous variables expressed as median (interquartile range) and $x^{2}$ tests for categorical variables expressed as count and percent 
Table 1 (continued)

SOFA Sequential organ failure score, $P C I S$ Pediatric critical illness score, MODS Multiple organ dysfunction syndrome, MOF Multiple organ failure, DIC Disseminated intravascular coagulation, ARDS Acute respiratory distress syndrome, TRIG Triglyceride, HDL High-density lipoprotein, LDL Low density lipoprotein, ALT Alanine transaminase, AST Aspartate aminotransferase, LD Lactate dehydrogenase, HBDH Hydroxybutyrate dehydrogenase, CK Creatine Kinase, CKMB Creatine phosphokinase-Mb, WBC White blood cell, CRP C-Reactive Protein, PCT Procalcitonin

Table 2 The comparisons of circulating biomarkers between children with sepsis and controls, as well as between survived and deceased in children with sepsis

\begin{tabular}{|c|c|c|c|c|c|c|}
\hline \multirow[t]{2}{*}{ Biomarkers } & \multirow[t]{2}{*}{ Controls } & \multicolumn{3}{|l|}{ Children with sepsis } & \multirow[t]{2}{*}{$P_{1}$} & \multirow[t]{2}{*}{$\mathrm{P}_{2}$} \\
\hline & & All & Deceased & Survived & & \\
\hline CoQ10 ( $\mu \mathrm{mol} / \mathrm{L})$ & 1.0 (0.8 to 1.2$)$ & $0.7(0.5$ to 1.0$)$ & 0.5 (0.4 to 0.6$)$ & $0.8(0.6$ to 1.1$)$ & $<0.001$ & $<0.001$ \\
\hline Complex I (nmol/min.mg) & 107.7 (95.2 to 120.8) & 107.1 (98.8 to 124.4) & 113.8 (98.5 to 125.0) & 106.2 (98.8 to 124.4) & 0.460 & 0.371 \\
\hline Complex II (nmol/min.mg) & 99.6 (88.0 to 108.1) & 88.2 (78.8 to 100.7) & 87.1 (70.1 to 98.5$)$ & 91.3 (79.9 to 101.4) & 0.004 & 0.499 \\
\hline Complex II + III (nmol/min.mg) & 186.3 (170.2 to 202.7) & 184.9 (158.8 to 199.8$)$ & $186.8(132.2$ to 207.5$)$ & 182.3 (163.6 to 199.6$)$ & 0.260 & 0.606 \\
\hline Complex IV (nmol/min.mg) & 136.3 (123.1 to 155.7$)$ & 143.6 (88.1 to 166.6$)$ & 149.2 (88.8 to 164.1$)$ & 132.3 (88.1 to 166.8) & 0.521 & 0.983 \\
\hline Complex I + III (nmol/min.mg) & 898.1 (806.9 to 1083.1) & 622.8 (384.7 to 811.9) & 236.8 (132.6 to 592.5$)$ & 731.7 (563.4 to 823.4) & $<0.001$ & $<0.001$ \\
\hline FoF1-ATPase (nmol/min.mg) & 751.9 (605 to 903.3) & 356.6 (222.8 to 544.7$)$ & 383.9 (215.3 to 710.8$)$ & $335.2(222.8$ to 535.1$)$ & $<0.001$ & 0.486 \\
\hline
\end{tabular}

$P$ values are calculated by t-test for normal variables and nonparametric Wilcoxon rank sum tests for non-normal data expressed as median (interquartile range). $P_{1}$ : Children with sepsis versus controls; $\mathrm{P}_{2}$ : Survived versus deceased

CoQ10 Coenzyme Q10

Table 3 Risk prediction of circulating biomarkers for the risk of sepsis, as well as the mortality risk

\begin{tabular}{|c|c|c|c|c|c|c|}
\hline Significant risk factors & COR & $95 \% \mathrm{Cl}$ & $\mathbf{P}$ & aOR & $95 \% \mathrm{Cl}$ & $\mathbf{P}^{*}$ \\
\hline \multicolumn{7}{|l|}{ Children with sepsis versus controls } \\
\hline CoQ10 (+ $0.05 \mu \mathrm{mol} / \mathrm{L})$ & 0.88 & 0.82 to 0.95 & 0.001 & 0.85 & 0.77 to 0.93 & 0.001 \\
\hline Complex I (+20 nmol/min.mg) & 1.19 & 0.75 to 1.89 & 0.456 & 1.02 & 0.59 to 1.78 & 0.942 \\
\hline Complex II (+50 nmol/min.mg) & 0.29 & 0.10 to 0.85 & 0.025 & 0.29 & 0.10 to 0.85 & 0.025 \\
\hline Complex II + III (+50 nmol/min.mg) & 0.71 & 0.39 to 1.28 & 0.258 & 0.42 & 0.20 to 0.90 & 0.026 \\
\hline Complex IV (+ 100 nmol/min.mg) & 0.69 & 0.23 to 2.10 & 0.517 & 0.25 & 0.06 to 1.04 & 0.057 \\
\hline Complex I + III (+50 nmol/min.mg) & 0.71 & 0.61 to 0.83 & $<0.001$ & 0.68 & 0.57 to 0.82 & $<0.001$ \\
\hline FoF1-ATPase (+ 100 nmol/min.mg) & 0.53 & 0.42 to 0.68 & $<0.001$ & 0.53 & 0.40 to 0.70 & $<0.001$ \\
\hline \multicolumn{7}{|c|}{ Children with sepsis: deceased versus survived } \\
\hline CoQ10 (+0.05 $\mu \mathrm{mol} / \mathrm{L})$ & 0.74 & 0.61 to 0.90 & 0.003 & 0.73 & 0.59 to 0.90 & 0.004 \\
\hline Complex I (+ 20 nmol/min.mg) & 1.35 & 0.71 to 2.58 & 0.364 & 1.38 & 0.71 to 2.65 & 0.340 \\
\hline Complex II (+ 50 nmol/min.mg) & 1.30 & 0.36 to 4.80 & 0.690 & 1.32 & 0.35 to 4.96 & 0.678 \\
\hline Complex I+ III (+50 nmol/min.mg) & 0.81 & 0.37 to 1.77 & 0.598 & 0.79 & 0.33 to 1.85 & 0.580 \\
\hline Complex IV (+ 100 nmol/min.mg) & 1.15 & 0.29 to 4.59 & 0.841 & 1.14 & 0.27 to 4.80 & 0.857 \\
\hline Complex I + III (+50 nmol/min.mg) & 0.79 & 0.68 to 0.91 & 0.001 & 0.76 & 0.64 to 0.89 & 0.001 \\
\hline FoF1-ATPase (+ 100 nmol/min.mg) & 1.08 & 0.87 to 1.35 & 0.478 & 1.08 & 0.87 to 1.35 & 0.481 \\
\hline
\end{tabular}

COR crude odds ratio, aOR adjusted odds ratio, 95\% Cl 95\% confidence interval

*P was calculated after adjusting for age and sex. Data are expressed as odds ratio, $95 \%$ confidence interval, P value

mitochondrial dysfunction plays a contributory role in the development of sepsis, especially the abnormal expressions of mitochondrial respiratory chain complexes and mobile carriers. In support of this hypothesis, Donnino and colleagues conducted a randomized, double-blind, placebo-controlled, pilot trial, by showing that plasma CoQ10 levels were increased in patients with severe sepsis or septic shock, with the administration of oral ubiquinol [35]. However, the contribution of mitochondrial respiratory chain complexes and mobile carriers to the development and progression of sepsis has been rarely reported. To shed some light, we assayed the activities of mitochondrial respiratory chain enzymes in circulation among children with and 
Table 4 Prediction accuracy for sepsis mortality risk gained by adding each circulating biomarker to basic model

\begin{tabular}{|c|c|c|c|c|c|c|c|c|c|}
\hline \multirow[t]{2}{*}{ Statistics } & \multirow[t]{2}{*}{ Basic Model } & \multicolumn{8}{|c|}{ Basic Model Plus } \\
\hline & & CoQ10 & Complex I & $\begin{array}{l}\text { Complex } \\
\text { II+ III }\end{array}$ & $\begin{array}{l}\text { Complex } \\
\text { II+ III }\end{array}$ & Complex IV & Complex I+ III & FoF1-ATPase & $\begin{array}{l}\text { CoQ10 and } \\
\text { Complex I } \\
+ \text { III }\end{array}$ \\
\hline \multicolumn{10}{|l|}{ Calibration } \\
\hline $\mathrm{AlC}$ & 56.67 & 44.57 & 50.70 & 49.36 & 49.04 & 50.49 & 43.06 & 49.84 & 39.96 \\
\hline $\mathrm{BIC}$ & 68.14 & 57.95 & 64.08 & 62.74 & 62.43 & 63.87 & 56.44 & 63.23 & 55.25 \\
\hline$L R$ & Ref & 14.1 & 7.97 & 9.31 & 9.62 & 8.18 & 15.61 & 8.82 & 20.71 \\
\hline$L R(P)$ & Ref & $<0.001$ & 0.005 & 0.002 & 0.002 & 0.004 & $<0.001$ & 0.003 & $<0.001$ \\
\hline \multicolumn{10}{|l|}{ Discrimination } \\
\hline$N R I$ & Ref & 0.032 & 0.317 & 0.083 & 0.157 & 0.498 & 0.046 & 0.632 & 0.005 \\
\hline $\mid \mathrm{DI}$ & Ref & 0.003 & 0.808 & 0.265 & 0.575 & 0.392 & 0.006 & 0.377 & $<0.001$ \\
\hline ROC area & 0.881 & 0.932 & 0.881 & 0.884 & 0.893 & 0.888 & 0.928 & 0.903 & 0.950 \\
\hline ROC curve $P$ & Ref & 0.143 & 0.389 & 0.864 & 0.593 & 0.731 & 0.185 & 0.613 & 0.047 \\
\hline
\end{tabular}

AIC Akaike information criterion, $B I C$ Bayesian information criterion, $L R$ likelihood ratio, NRI net reclassification improvement, $I D I$ integrated discrimination improvement, ROC Relative operating characteristic curve, Ref. reference group. Basic model included age, gender, Pediatric critical illness score (Day 1 ), number of multiple organ failure, and shock

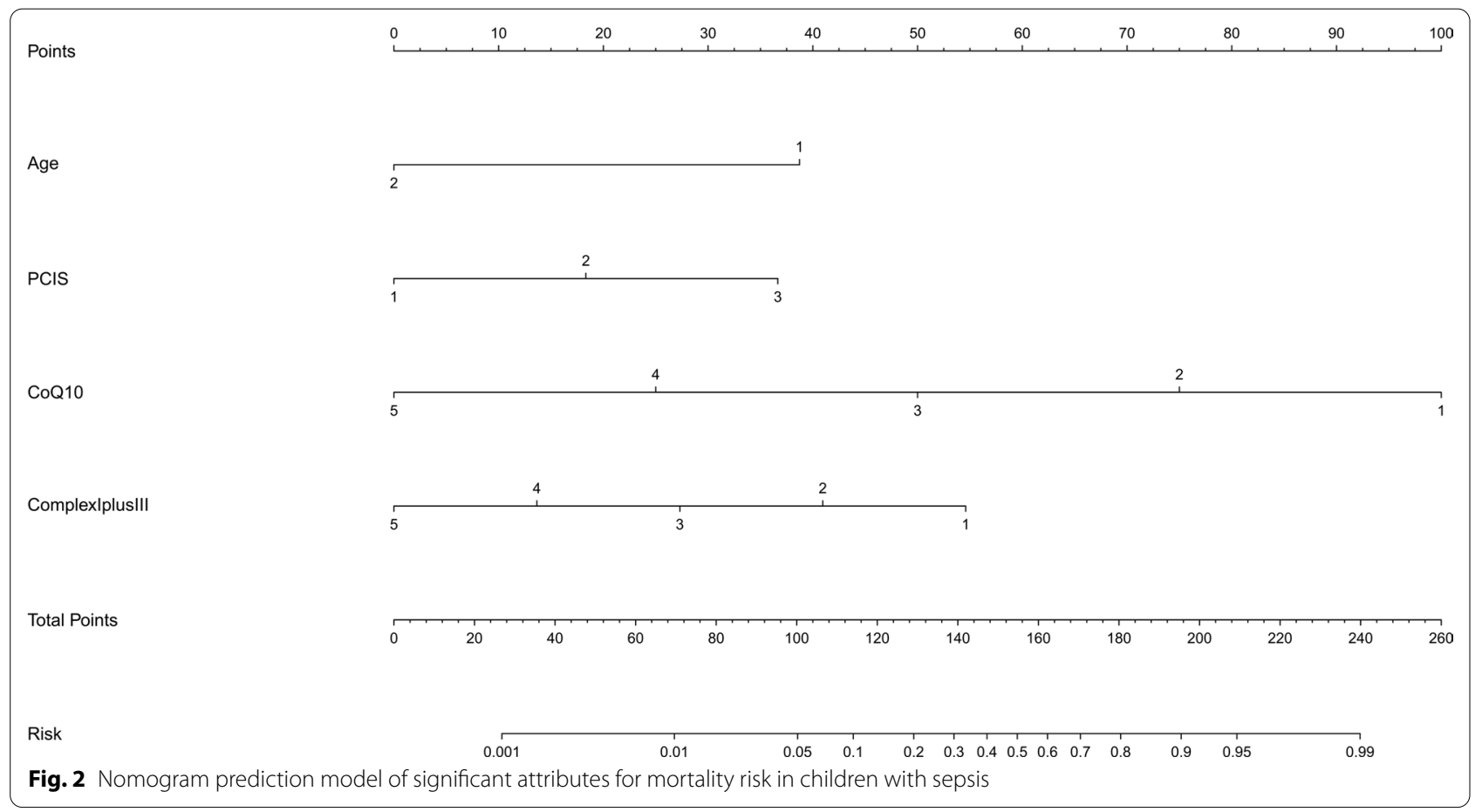

without clinically-confirmed sepsis to examine their association with the risk and mortality of pediatric sepsis.

After a comprehensive analysis, we interestingly found that high CoQ10 and complex I+ III levels were significantly associated with the reduced risk of having pediatric sepsis, as well as the reduced risk of dying from sepsis during hospitalization. CoQ10 has been proposed as an effective agent for reducing the deleterious effects of septic shock by acting as an oxygen free radical scavenger and thus stabilizing mitochondrial membranes, as well as by inhibiting the arachidonic acid metabolic pathway and the formation of various prostaglandins. There is evidence that CoQ10 is effective in alleviating histological organ damage in sepsis via mortality statistics of mice model [36]. In addition, animal studies indicated that complex I+ III activity was higher in the sepsis groups 
than healthy controls in septic mice models caused by lipopolysaccharide, and after treatment with Simvastatin, mitochondrial complex I+ III expression was increased [37]. Given the significant association observed in this present study and strong biological implications, it would be tempting to speculate that dysregulation of mitochondrial respiratory chain, in particular CoQ10 and complex I+ III, is attributable to the pathogenesis of pediatric sepsis. Moreover, considering the high mortality rate of sepsis in children, identification of circulating biomarkers is of great importance in sanitary science and public health.

Although CoQ10 cannot be bedside-tested at present, it can be administered exogenously to improve the low-level of CoQ10 in septic children. Currently, oral CoQ 10 has been reported to inhibit the inflammatory response in migraine in a randomized doubleblind placebo-controlled clinical trial [38]. Meanwhile, due to the important role of CoQ10 in the mitochondrial electron transport chain oxidation respiratory chain, it has shown useful effects in other interventional experiments such as polycystic ovary syndrome, fibromyalgia et al. $[39,40]$. Based on the available evidence, it is reasonable to believe that CoQ10 opens the potential for future therapeutic interventions in septic children. In addition, to improve the serum CoQ10 by supplementing appropriate vitamins B2, B9, B12, and $C$ are all possible to improve pediatric sepsis by maintaining mitochondrial function [41]. Additional research are worth designing to investigate the potential of CoQ10 as a therapeutic agent in children with sepsis.

Several limitations should be acknowledged for this study. First, the small sample size involved may limit the power to detect small contributions. Second, all study participants are of Chinese descent, which may limit the extrapolation of our findings to other ethnic groups. Third, only a death-or-discharge outcome was recorded during hospitalization and further followup evaluation was not available for us. At the same time, this research lack laboratory indicators for the healthy group. Fourth, mitochondrial respiratory chain enzyme activities were assayed only once, and their dynamic monitoring is of added interest.

Taken together, our findings indicate the promising predictive contribution of low serum CoQ10 and complex I+III to the risk of pediatric sepsis and its associated mortality during hospitalization among Chinese children. Given the aforementioned limitations, we agree that further investigations on the molecular mechanisms linking mitochondrial respiratory chain enzymes and pediatric sepsis are warranted.

\section{Conclusion}

Low serum CoQ10 and decreased mitochondrial complex I+ III activity can predict the sepsis incidence and related mortality among Chinese children.

\begin{abstract}
Abbreviations
CoQ10: Coenzyme Q10; PICU: Pediatric Intensive Care Unit; SOFA: Sequential organ failure score; PCIS: Pediatric critical illness score; ARDS: Acute respiratory distress syndrome; LD: Lactate dehydrogenase; $\mathrm{HBDH}$ : Hydroxybutyrate dehydrogenase; AIC: Akaike information criterion; BIC: Bayesian information criterion; NRI: Net reclassification improvement; IDI: Integrated discrimination improvement; ROC: Receiver operating characteristic; OR: Odds ratio; $\mathrm{Cl}$ : Confidence interval; $P$ : Inconsistency index.
\end{abstract}

\section{Supplementary Information}

The online version contains supplementary material available at https://doi. org/10.1186/s12879-021-07014-6.

Additional file 1: eFile S1. The STROBE checklist.

Additional file 2: eFile S2. Definitions of systemic inflammatory response syndrome (SIRS), infection, sepsis.

\section{Acknowledgements}

We are grateful to all participating patients for their cooperation and willingness.

\section{Authors' contributions}

QZ planned and designed the study, and directed its implementation. QZ drafted the protocol. NL, LZ, and XL obtained statutory and ethics approvals. $\mathrm{DH}, \mathrm{NL}$, and XC contributed to data acquisition. DH, YC, LG, and WN conducted statistical analyses. DH, ZS, WL, XL and GS did the data preparation and quality control. DH, WN, LZ and QZ wrote the manuscript. All authors read and approved the final manuscript.

\section{Funding}

This work was financially supported by the Clinical Technology Innovation Project of Beijing Municipal Administration of Hospitals (Grant No. XM201412), and the Beijing Natural Science Foundation (Grant No. 7172037). The funder had no role in any other part of the trial (including study design, data collection, and data interpretation et al.) or writing the manuscript.

\section{Availability of data and materials}

The datasets used and/or analysed during the current study are available from the corresponding author on reasonable request.

\section{Declarations}

Ethics approval and consent to participate

Our study design received approval from ethics committee of Children's Hospital Affiliated to the Capital Institute of Pediatrics. Written informed consent was obtained from a parent or guardian for all study participants. The authors are accountable for all aspects of the work in ensuring that questions related to the accuracy or integrity of any part of the work are appropriately investigated and resolved.

\section{Consent to publication}

Not applicable.

\section{Competing interests}

The authors declare no Competing Interest.

\section{Author details}

${ }^{1}$ Institute of Clinical Medical Sciences, China-Japan Friendship Hospital, No. 2 Yinghua East Street, Chaoyang District, Beijing 100029, China. ${ }^{2}$ Department 
of Pediatrics, China-Japan Friendship Hospital, No.2 Yinghua East Street, Chaoyang District, Beijing 100029, China. Intensive Care Unit, Affiliated Children's Hospital of Capital Institute of Pediatrics, Beijing, China. ${ }^{4}$ Graduate School of Peking Union Medical College and Chinese Academy of Medical Science, Beijing, China. ${ }^{5}$ Central Laboratory, Capital Institute of Pediatrics, Beijing, China.

Received: 1 September 2020 Accepted: 23 December 2021 Published online: 06 January 2022

\section{References}

1. Tan B, Wong JJ, Sultana R, Koh J, Jit M, Mok YH, Lee JH. Global case-fatality rates in pediatric severe sepsis and septic shock: a systematic review and meta-analysis. JAMA Pediatr. 2019;173(4):352-62.

2. Weiss SL, Nicolson SC, Naim MY. Clinical update in pediatric sepsis: focus on children with pre-existing heart disease. J Cardiothorac Vasc Anesth. 2019;34(5):1324-32.

3. Wang Y, Sun B, Yue H, Lin X, Li B, Yang X, Shan C, Fan Y, Dong M, Zhang Y, et al. An epidemiologic survey of pediatric sepsis in regional hospitals in China. Pediatric Crit Care Med. 2014;15(9):814-20.

4. Organization GWH: Seventieth World Health Assembly update [news release]: www.whoint/mediacentre/news/releases/2017/wha70/en/ .Accessed 2018 Mar 2017.

5. Mikkelsen ME, Miltiades AN, Gaieski DF, Goyal M, Fuchs BD, Shah CV Bellamy SL, Christie JD. Serum lactate is associated with mortality in severe sepsis independent of organ failure and shock. Crit Care Med. 2009;37(5):1670-7.

6. Fitrolaki MD, Dimitriou H, Venihaki M, Katrinaki M, Ilia S, Briassoulis G. Increased extracellular heat shock protein 90a in severe sepsis and SIRS associated with multiple organ failure and related to acute inflammatorymetabolic stress response in children. Medicine. 2016;95(35):e4651.

7. Vardas K, Ilia S, Sertedaki A, Charmandari E, Briassouli E, Goukos D, Apostolou K, Psarra K, Botoula E, Tsagarakis S, et al. Increased glucocorticoid receptor expression in sepsis is related to heat shock proteins, cytokines, and cortisol and is associated with increased mortality. Intensive Care Med Exp. 2017;5(1):10.

8. Ilia S, Briassoulis G. Sepsis is change and flows ever onwards. Expert Rev Anti Infect Ther. 2017;15(6):515-7.

9. Briassoulis G, Briassoulis P, Miliaraki M, Ilia S, Parlato M, Philippart F, Rouquette A, Moucadel V, Cavaillon JM, Misset B. Biomarker cruises in sepsis: who is the CAPTAIN? Discussion on "Circulating biomarkers may be unable to detect infection at the early phase of sepsis in ICU patients: the CAPTAIN prospective multicenter cohort study." Intensive Care Med. 2019;45(1):132-3.

10. Beloborodova N, Pautova A, Sergeev A, Fedotcheva N. Serum levels of mitochondrial and microbial metabolites reflect mitochondrial dysfunction in different stages of sepsis. Metabolites. 2019;9:10.

11. Colunga Biancatelli RML, Berrill M, Mohammed YH, Marik PE. Melatonin for the treatment of sepsis: the scientific rationale. J Thorac Dis. 2020;12(Suppl 1):S54-65.

12. Manfredini A, Constantino L, Pinto MC, Michels M, Burger H, Kist LW, Silva MC, Gomes LM, Dominguini D, Steckert A, et al. Mitochondrial dysfunction is associated with long-term cognitive impairment in an animal sepsis model. Clin Sci (Lond). 2019;133(18):1993-2004.

13. Weiss SL, Zhang D, Bush J, Graham K, Starr J, Tuluc F, Henrickson S, Kilbaugh T, Deutschman CS, Murdock D, et al. Persistent mitochondrial dysfunction linked to prolonged organ dysfunction in pediatric sepsis. Crit Care Med. 2019;47(10):1433-41.

14. Sun J, Zhang J, Tian J, Virzi GM, Digvijay K, Cueto L, Yin Y, Rosner MH, Ronco C. Mitochondria in Sepsis-Induced AKI. J Am Soc Nephrol. 2019;30(7):1151-61.

15. Sureshbabu A, Patino E, Ma KC, Laursen K, Finkelsztein EJ, Akchurin O, Muthukumar T, Ryter SW, Gudas L, Choi AMK, et al. RIPK3 promotes sepsis-induced acute kidney injury via mitochondrial dysfunction. JCI insight. 2018;3:11.

16. Inata Y, Kikuchi S, Samraj RS, Hake PW, O'Connor M, Ledford JR, O'Connor J, Lahni P, Wolfe V, Piraino G, et al. Autophagy and mitochondrial biogenesis impairment contribute to age-dependent liver injury in experimental sepsis: dysregulation of AMP-activated protein kinase pathway. FASEB J. 2018;32(2):728-41.

17. Pinto BB, Dyson A, Umbrello M, Carre JE, Ritter C, Clatworthy I, Duchen MR, Singer M. Improved survival in a long-term rat model of sepsis is associated with reduced mitochondrial calcium uptake despite increased energetic demand. Crit Care Med. 2017;45(8):e840-8.

18. Samuels DC, Hulgan T, Fessel JP. Mitochondrial DNA Haplogroups and delirium during sepsis. Crit Care Med. 2019;47(8):1065-71.

19. Xin T, Lu C. SirT3 activates AMPK-related mitochondrial biogenesis and ameliorates sepsis-induced myocardial injury. Aging. 2020;12(16):16224-37.

20. Goldstein B, Giroir B, Randolph A. International pediatric sepsis consensus conference: definitions for sepsis and organ dysfunction in pediatrics. Pediatric Crit Care Med. 2005;6(1):2-8.

21. Tang PH, Miles MV, DeGrauw A, Hershey A, Pesce A. HPLC analysis of reduced and oxidized coenzyme Q(10) in human plasma. Clin Chem. 2001;47(2):256-65.

22. Prilutskiĭ AS, Khodakovskil̆ AV, Mălian EA. A method of separating mononuclears on a density gradient. Lab Delo. 1990;2:20-3.

23. Bradford MM. A rapid and sensitive method for the quantitation of microgram quantities of protein utilizing the principle of protein-dye binding. Anal Biochem. 1976;72:248-54.

24. Rustin P, Chretien D, Bourgeron T, Gérard B, Rötig A, Saudubray JM, Munnich A. Biochemical and molecular investigations in respiratory chain deficiencies. Clinica Chimica Acta. 1994;228(1):35-51.

25. Miró O, Cardellach F, Barrientos A, Casademont J, Rötig A, Rustin P. Cytochrome c oxidase assay in minute amounts of human skeletal muscle using single wavelength spectrophotometers. J Neurosci Methods. 1998:80(1):107-11.

26. Chretien D, Bénit P, Chol M, Lebon S, Rötig A, Munnich A, Rustin P. Assay of mitochondrial respiratory chain complex I in human lymphocytes and cultured skin fibroblasts. Biochem Biophys Res Commun. 2003;301(1):222-4.

27. Ternes N, Rotolo F, Michiels S. Empirical extensions of the lasso penalty to reduce the false discovery rate in high-dimensional Cox regression models. Stat Med. 2016;35(15):2561-73.

28. Pencina MJ, D'Agostino RB, D'Agostino RB, Jr., Vasan RS: Evaluating the added predictive ability of a new marker: from area under the ROC curve to reclassification and beyond. Statistics in medicine 2008, 27(2):157-172. (discussion 207-112).

29. Pencina MJ, D'Agostino RB, Vasan RS. Statistical methods for assessment of added usefulness of new biomarkers. Clin Chem Lab Med. 2010;48(12):1703-11.

30. Beam CA. Analysis of clustered data in receiver operating characteristic studies. Stat Methods Med Res. 1998;7(4):324-36.

31. Chen H, Mao X, Meng X, Li Y, Feng J, Zhang L, Zhang Y, Wang Y, Yu Y, Xie K. Hydrogen alleviates mitochondrial dysfunction and organ damage via autophagymediated NLRP3 inflammasome inactivation in sepsis. Int J Mol Med. 2019;44(4):1309-24

32. Chen G, Li X, Huang M, Zhou X, Li Y, Mao X, Bai J. The role of thioredoxin-1 in suppression sepsis through inhibiting mitochondrial-induced apoptosis in spleen. Shock. 2017;47(6):753-8.

33. Kohoutova M, Dejmek J, Tuma Z, Kuncova J. Variability of mitochondrial respiration in relation to sepsis-induced multiple organ dysfunction. Physiol Res. 2018;67(Suppl 4):S577-s592.

34. Singer M. Mitochondrial function in sepsis: acute phase versus multiple organ failure. Crit Care Med. 2007;35(9 Suppl):S441-448.

35. Donnino MW, Mortensen SJ, Andersen LW, Chase M, Berg KM, Balkema J, Radhakrishnan J, Gazmuri RJ, Liu X, Cocchi MN. Ubiquinol (reduced Coenzyme Q10) in patients with severe sepsis or septic shock: a randomized, double-blind, placebo-controlled, pilot trial. Crit Care (London, England). 2015;19:275.

36. Abitagaoglu S, Akinci SB, Saricaoglu F, Akinci M, Zeybek ND, Muftuoglu S, Aypar U. Effect of coenzyme Q10 on organ damage in sepsis. Bratisl Lek Listy. 2015;116(7):433-9.

37. Ozkok E, Yorulmaz H, Ates G, Serdaroglu-Oflazer P, Tamer AS. Effects of prior treatment with simvastatin on skeletal muscle structure and mitochondrial enzyme activities during early phases of sepsis. Int J Clin Exp Pathol. 2014;7(12):8356-65.

38. Dahri M, Tarighat-Esfanjani A, Asghari-Jafarabadi M, Hashemilar M. Oral coenzyme Q10 supplementation in patients with migraine: 
Effects on clinical features and inflammatory markers. Nutr Neurosci. 2019;22(9):607-15.

39. Liu M, Zhu H, Hu X, Zhu Y, Chen H. Efficacy of coenzyme Q10 supplementation on glucose metabolism, lipid profiles, and biomarkers of inflammation in women with polycystic ovary syndrome: A protocol for a systematic review and meta-analysis. Medicine. 2020;99(46):e23130.

40. Pagliai G, Giangrandi I, Dinu M, Sofi F, Colombini B. Nutritional Interventions in the Management of Fibromyalgia Syndrome. Nutrients. 2020;12:9.

41. Can A, Temel M, Dokuyucu R, Mutaf M. The effect of coenzyme Q10 (Ubiquinone) on random pattern skin flap survival in rat model. Ann Plast Surg. 2016;77(2):e9-14.

\section{Publisher's Note}

Springer Nature remains neutral with regard to jurisdictional claims in published maps and institutional affiliations.

- fast, convenient online submission

- thorough peer review by experienced researchers in your field

- rapid publication on acceptance

- support for research data, including large and complex data types

- gold Open Access which fosters wider collaboration and increased citations

- maximum visibility for your research: over $100 \mathrm{M}$ website views per year

At BMC, research is always in progress.

Learn more biomedcentral.com/submissions 